\title{
A Novel Mri-Method Allows a Rapid and Robust Assessment of Muscle Quantity and Quality in Hemodialysis Patients.
}

\author{
Ainhoa Indurain ( $\nabla$ ainhoa.indurain@liu.se ) \\ Linköping University Hospital \\ Jennifer Linge \\ AMRA Medical AB \\ Mikael Petersson Petersson \\ AMRA Medical AB
}

\section{Thobias Romu}

AMRA Medical AB

\section{Fredrik Uhlin}

Linköping University Hospital

\section{Anders Fernström}

Linköping University Hospital

\section{Mårten Segelmark}

Lund University

Olof Dahlqvist Leinhard

Linköping University Hospital

\section{Research Article}

Keywords: Chronic kidney disease, Comorbidity, Hemodialysis, Magnetic Resonance Imaging, Mortality, Sarcopenia

Posted Date: December 29th, 2021

DOI: https://doi.org/10.21203/rs.3.rs-1111144/v1

License: (c) (i) This work is licensed under a Creative Commons Attribution 4.0 International License. Read Full License 


\section{Abstract}

\section{Background}

Sarcopenia is a prevalent condition in patients on maintenance hemodialysis and associates with mortality. Research using magnetic resonance imaging (MRI) has demonstrated the importance

of a proper body size-adjustment in the assessment of muscle mass, and that a muscle composition assessment including measurements of both muscle volume and fat infiltration improves the prediction of comorbidity and survival related to sarcopenia. Such combined muscle composition assessment has not previously been performed in hemodialysis patients.

\section{Methods}

Eleven hemodialysis patients were scanned using whole-body fat and water separated MRI and followed regarding survival and comorbidity for five years. Muscle composition was assessed using AMRA ${ }^{8}$ Researcher. Using data from 9615 UK Biobank participants, sex and BMI-matched muscle volume zscores and sex-adjusted muscle fat infiltration values were calculated for each patient. These measurements were then used for the calculation of a combined muscle score. Resulting three muscle measurements were associated with survival and comorbidity index.

\section{Results}

Six patients exhibited low muscle volume (muscle volume $z$-score $<25^{\text {th }}$ percentile (population wide)), and six patients presented with high muscle fat infiltration $\left(>75^{\text {th }}\right.$ percentile (population wide)). During a $68-$ months follow-up, five patients died, four were transplanted and two remained on hemodialysis treatment. The combined muscle score was significantly associated with comorbidity index $(p<0.05)$ and was highly predictive of survival (AUROC 0.97).

\section{Conclusions}

The combined assessment including both body size-adjusted muscle volume and muscle fat infiltration can be used to analyze muscle composition in hemodialysis patients. MRI based muscle composition assessment reflected comorbidity and predicted survival in hemodialysis patients.

\section{Background}

Patients with chronical kidney diseases (CKD) has a degenerative muscle process associated with both protein wasting (with higher muscle degradation) and decrease of muscle synthesis (1). This leads to a loss of muscle mass, strength and function and make them more predisposed to sarcopenia. Although sarcopenia is a slow and age-related process, a chronic disease state such as CKD may lead to more rapid decline in muscle mass and function (2). 
The relationship between increased prevalence of sarcopenia and progression of CKD is well described and is even more evident in patients with end stage kidney disease, especially when on dialysis treatment $(3,4)$. Furthermore, sarcopenia in CKD patients is associated with a poor quality of life, increased morbidity, hospitalization and mortality $(5,6)$.

The prevalence of sarcopenia in dialysis patients varies between 9.5 to $37.1 \%$ (7). This variation could be explained by differences between methods and cut-offs criteria used for sarcopenia assessment. A recent study using MRI has demonstrated that proper body size-adjustment is pivotal for identifying sarcopenia using muscle mass (8).

Muscle mass can be estimated by a variety of techniques, but MRI is considered a gold standard method (9). A standardized 6-minute MRI examination enables assessment of muscle volume and muscle fat infiltration (MFI) (10). MFI provides information of muscle quality and associates with low function, adverse outcomes, and mortality $(2,11)$. The combined observation of low muscle volume and high MFI, adverse muscle composition (adverse $M C$ ), has been shown to improve functional correlations and predict hospitalization (8). Recently, adverse MC was linked to poor function and metabolic comorbidities within subjects with non-alcoholic fatty liver disease and all-cause mortality in general population (12, 13).

In this study, hemodialysis (HD) patients were scanned using MRI and their muscle composition was assessed to investigate its association with comorbidity and mortality.

\section{Methods}

\section{STUDY SUBJECTS AND CLINICAL DATA}

In this prospective observational study, 11 adult patients undergoing HD at the University Hospital of Linköping were recruited between March-April 2014. The exclusion criteria were age less than 18 years and presence of any metallic implant. The Regional Ethical Review Board in Linköping approved (2013/475-31) the study protocol, and written informed consent was obtained from all patients.

Comorbidity score was assessed using the new comorbidity index $(\mathrm{nCl})(14,15)$.

Electronic health care records were used to calculate the nCland to retrieve the five-years survival data.

\section{MEASUREMENTS OF BODY COMPOSITION BY MRI}

Patients were scanned using rapid whole-body fat and water separated MRI. Fat-free muscle volume(FFMV) in the thighs and muscle fat infiltration (MFI) in anterior thighs were assessed using AMRA $®$ Researcher (AMRA Medical, Linköping Sweden) (10). For the calculations, normative data from 9615 subjects in UK Biobank was used (accessed under data access application 6569). 
FFMV: Fat-free muscle volume, the 'viable muscle tissue' (volume of all voxels with fat fraction $<50 \%$ ) in the thighs.

FFMVZ-score:Fat-free muscle volume z-score. For each patient, a matched virtual control group (VCG) was stratified among the participants from UK Biobank with complete muscle composition data. Each patient's VCG included at least 150 individuals with the same sex and similar BMI. Based on each VCG, a personalized muscle volume z-score was calculated measuring how many standard deviations each patient was from the mean thigh FFMV/height ${ }^{2}$ of their VCG. This variable is sex-, weight-, and height invariant and has been associated with poor function and increased hospitalization (8).

MFI: Muscle fat infiltration, the mean fat fraction within the 'viable muscle tissue' (FFMV) of the right and left anterior thighs. Due to the difference in magnitude between females (higher) and males (lower), a sex-adjusted MFI, MFIadj was calculated by subtracting the sex-specific population median in the UK Biobank dataset from MFI.

Muscle comb $_{\text {: }}$ Combined muscle score, was estimated projecting $M F I_{a d j}$ and FFMV z-score on the linear regression line describing the normal population relationship between $M F I_{a d j}$ and $F F M V Z$-score in the UK Biobank dataset.

\section{STATISTICAL ANALYSES}

Data was statistically analyzed using R.The Spearman rank correlation test was used to analyze the associations between muscle composition variables and comorbidity. To analyze the association between muscle composition variables and mortality, the Wilcoxon rank sum test and ROC analysis were used.

\section{Results}

A total of 11 patients were enrolled in the study. The majority were male with a mean age of $60.3 \pm 12.3$ years (Table 1$)$.

Table 1. Characteristics of the study subjects $n=11$. 


\begin{tabular}{|c|c|c|c|c|}
\hline Parameters & $\begin{array}{l}\text { All Patients } \\
(n=11)\end{array}$ & $\begin{array}{l}\text { Survivor } \\
\text { Group } \\
(n=6)\end{array}$ & $\begin{array}{l}\text { Non-survivor } \\
\text { Group } \\
(n=5)\end{array}$ & $\begin{array}{l}P \text {-value survivor vs } \\
\text { non-survivor }\end{array}$ \\
\hline Age, years & $60.3 \pm 12.3$ & $59.3 \pm 14.3$ & $61.4 \pm 10.9$ & 0.792 \\
\hline Gender (M/F) & $9 / 2$ & $4 / 2$ & $5 / 0$ & \\
\hline Body Mass Index, $\mathrm{kg} / \mathrm{m}^{2}$ & $26.7 \pm 4.8$ & $26.8 \pm 3.4$ & $26.5 \pm 6.5$ & 0.931 \\
\hline \multicolumn{5}{|l|}{ MRI Data } \\
\hline $\begin{array}{l}\text { Fat-free thigh muscle } \\
\text { volume, } L\end{array}$ & $11.1 \pm 2.7$ & $11.6 \pm 2.4$ & $10.6 \pm 3.2$ & 0.537 \\
\hline $\begin{array}{l}\text { Fat-free muscle volume } \\
\text { index, } \mathrm{L} / \mathrm{m}^{2}\end{array}$ & $3.5 \pm 0.7$ & $3.8 \pm 0.6$ & $3.2 \pm 0.7$ & 0.177 \\
\hline Muscle volume z-score, SD & $-0.8 \pm 1.4$ & $0.1 \pm 0.9$ & $-1.9 \pm 1.1$ & 0.017 \\
\hline Muscle fat infiltration, \% & $9.8 \pm 3.9$ & $7.3 \pm 1.6$ & $12.7 \pm 3.8$ & 0.017 \\
\hline $\mathrm{MFI}_{\text {adj: }}$ MFI Adjusted & $3.0 \pm 4.0$ & $0.4 \pm 1.5$ & $6.1 \pm 3.8$ & 0.017 \\
\hline \multicolumn{5}{|l|}{ Laboratory examinations } \\
\hline Hemoglobin, $g / L$ & $117.0 \pm 13.1$ & $115.3 \pm 14.5$ & $119.2 \pm 11.5$ & 1.0 \\
\hline Hematocrit, \% & $35.5 \pm 4.4$ & $35.2 \pm 5.2$ & $36.0 \pm 3.7$ & 1.0 \\
\hline Phosphate, mmol/L & $1.6 \pm 0.3$ & $1.6 \pm 0.3$ & $1.6 \pm 0.3$ & 1.0 \\
\hline Albumin, g/L & $35.8 \pm 3.6$ & $38.3 \pm 1.9$ & $32.8 \pm 2.6$ & 0.010 \\
\hline Ferritin, $\mu g / L$ & $465.2 \pm 275.1$ & $492.8 \pm 198.3$ & $432.0 \pm 370.8$ & 0.931 \\
\hline \multicolumn{5}{|l|}{ Dialysis characteristics } \\
\hline $\begin{array}{l}\text { Dialysis mode (HD/OL- } \\
\text { HDF) }\end{array}$ & $7 / 4$ & $5 / 1$ & $2 / 3$ & \\
\hline Dialysis duration, months & $31.4 \pm 27.9$ & $40 \pm 31.8$ & $21 \pm 20.7$ & 0.200 \\
\hline Dialysis time/week, hours & $15.0 \pm 3.5$ & $14.7 \pm 2.1$ & $15.3 \pm 5.0$ & 0.706 \\
\hline $\mathrm{n}^{\text {AV-fistula/dialysis catheter, }}$ & $9 / 2$ & $5 / 1$ & $4 / 1$ & \\
\hline \multicolumn{5}{|l|}{$\begin{array}{l}\text { Causes of end-stage renal } \\
\text { diseases }\end{array}$} \\
\hline Diabetic nephropathy & 4 & 0 & 4 & \\
\hline Chronic glomerulonephritis & 3 & 2 & 1 & \\
\hline
\end{tabular}




\begin{tabular}{|c|c|c|c|}
\hline Polycystic kidney disease & 1 & 1 & 0 \\
\hline $\begin{array}{l}\text { Chronic infravesical } \\
\text { obstruction }\end{array}$ & 1 & 1 & 0 \\
\hline $\begin{array}{l}\text { Renal cancer/bilateral } \\
\text { nephrectomy }\end{array}$ & 1 & 1 & 0 \\
\hline Unknown & 1 & 1 & 0 \\
\hline \multicolumn{4}{|l|}{ Comorbidities, n (\%) } \\
\hline Congestive heart failure & $3(27)$ & $1(17)$ & $2(40)$ \\
\hline Coronary artery disease & $4(36)$ & 0 & $4(80)$ \\
\hline Cerebrovascular disease & $2(18)$ & 0 & $2(40)$ \\
\hline Diabetes mellitus & $4(36)$ & 0 & $4(80)$ \\
\hline $\begin{array}{l}\text { Peripheral vascular } \\
\text { disease }\end{array}$ & $4(36)$ & 0 & $4(80)$ \\
\hline
\end{tabular}

For continuous variables, mean and standard deviation are shown. $P$-value using the Wilcoxon rank sum test. HD: Hemodialysis. OL-HDF: On-Line Hemodiafiltration.

Seven patients exhibited lower muscle volume and eight patients higher MFI as compared with their matched controls (Fig 2). According to population wide cut-points for adverse $M C$, six patients presented with low muscle volume ( $<25^{\text {th }}$ percentile for FFMV z-score) and six patients with high MFI $\left(>75^{\text {th }}\right.$ percentile for MFI). A total of five patients presented with adverse $M C$ (low muscle volume coupled with high MFI).

The muscle volume z-score (FFMV z-score) and muscle fat measurement $\left(M F l_{a d j}\right)$ did not significantly

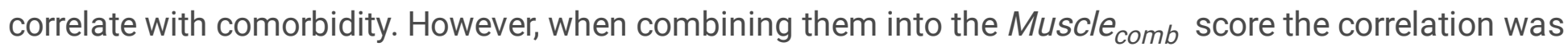
statistically significant (Table 2).

Table 2. Association between muscle composition variables and comorbidity.

\begin{tabular}{lll} 
& Estimate of rank correlation & $P$-value \\
\hline FFMV z-score & -0.5448 & 0.0831 \\
\hline $\mathrm{MFI}_{\text {adj }}$ & 0.4731 & 0.1416 \\
\hline Muscle $_{\text {comb }}$ & 0.6022 & 0.0499
\end{tabular}

Spearman rank correlation tests. FFMV z-score: virtual control group-based fat free muscle volume. $\mathrm{MFI}_{\text {adj: }}$ muscle fat infiltration adjusted for sex. Muscle $\mathrm{e}_{\mathrm{comb}}$ : combined measurement. 
During the follow-up period of 68 months, five of the 11 patients enrolled in the study died (two of cardiovascular disease, one of cerebrovascular disease, two of infection), four patients were kidney transplanted, and two patients remained on HD. Patients in the non-survivor group, when comparing with the survivor group, had significantly lower muscle volume z-score and higher MFI (Table 1).

The three muscle composition variables, FFMV z-score, $M F I_{a d j}$ and $M u s c l e_{c o m b}$, exhibited a significant positive correlation with mortality (Table 3 ). Furthermore, the numerically strongest association was found for the combined variable, Muscle comb.

\section{Table 3. Association between muscle composition variables and mortality}

\begin{tabular}{lllll} 
& Rank sum deaths & Minimum rank sum & Maximum rank sum & $P$-value \\
\hline FFMV z-score & 17 & 15 & 45 & 0.0173 \\
\hline $\mathrm{MFI}_{\text {adj }}$ & 43 & 15 & 45 & 0.0173 \\
\hline Muscle $_{\text {comb }}$ & 44 & 15 & 45 & 0.0087
\end{tabular}

Wilcoxon rank sum tests. FFMV z-score: virtual control group-based fat free muscle volume. $\mathrm{MFI}_{\text {adj: }}$ :

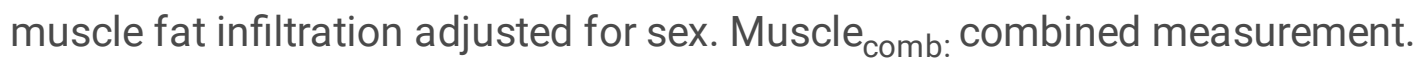

The area under ROC curves (AUROC) for predicting survival were 0.93 (0.78-1.00) for muscle volume (FFMV z-score), 0.93 (0.78-1.00) for the muscle fat measurement ( $\left.M F I_{a d j}\right)$ and 0.97 (0.87-1.00) for the combined muscle score (Muscle comb ).

A 2D-plot visualization of muscle volume (FFMV z-score) and sex-adjusted MFI ( $\left.M F I_{a d j}\right)$ showed that muscle composition was strongly associated with both comorbidity score and death (Fig 1).

\section{Discussion}

The present study demonstrates the feasibility of this MRI technique for assessment of muscle composition in HD patients and that combined assessment of muscle volume and muscle fat seems to predict mortality. Furthermore, the combination of these biomarkers, the combined variable Muscle comb, provides a more complete and muscle specific description.

The current consensus for evaluating sarcopenia recommends assessing muscle quantity as well as muscle function (strength or performance)(2). Due to the high correlation between muscle quantity and body size, the patient's body size needs to be considered when assessing if they have a proper amount of muscle quantity or no. This is commonly done by creating a skeletal muscle index - muscle quantity divided by height ${ }^{2}$, weight or BMI. However, the most appropriate method of indexing muscle quantity remains still uncertain $(8,16)$. Recently, Linge et al. used MRI in a study of sarcopenic obesity and reported that common ways to adjust muscle quantity for body size (division by height ${ }^{2}$, weight or BMI) 
does not effectively normalize this relationship and confounds studies on sarcopenia. They suggested a body size adjustment for muscle volume using personalized virtual control groups, that ultimately enabled BMI-independent sarcopenia assessment(8). The same method was applied in this study.

The etiology of sarcopenia in CKD patients is still unclear but multiple factors are involved. Conditions that are directly related to CKD pathogenesis, such as cardiovascular diseases and diabetes mellitus (DM), are also independently associated with sarcopenia(17-19). Therefore, we analyzed the relationship between muscle composition and comorbidity index $(\mathrm{nCl})$ in our patient cohort. We observed that the combined muscle score achieved by muscle volume z-score and MFI was significantly associated with the comorbidity index, but the association with the separate measurements did not reach significant level.

Our results suggest that the comorbidity score may identify patients who are more likely to have sarcopenia. Other studies have also found an association between $\mathrm{nCl}$ and sarcopenia supporting the idea that $\mathrm{nCl}$ could be used as a predictor to identify sarcopenia $(20,21)$.

As mentioned above, sarcopenia often co-exists with DM in HD patients and there is evidence that DM is an independent risk factor for the development of sarcopenia $(17,22)$. Interestingly, in our study, all patients with DM belonged to the non-survivor group where adverse $M C$ with low muscle volume $z$-score and high MFI were dominant.

Sarcopenia in CKD is associated with important clinical outcomes such as poor quality of life, cognitive impairment, depression, functional decline, frailty and hospitalization (5). Several studies have also reported that sarcopenia is an independent predictor of mortality in CKD patients (23). A new study on 9 000 individuals with CKD on UK Biobank reported that the presence of sarcopenia in CKD increased the mortality risk by $33 \%(24)$. In our study, we found that both muscle volume and MFI were significantly and independently associated with mortality and this association was even stronger when the combined score was assessed.

In line with our results, a study with patients undergoing hemodialysis reported that both low muscle mass and reduced muscle strength was associated with mortality (6). Interestingly, analysis of muscle composition data showed that adverse $M C$ (low muscle volume z-score coupled to high MFI) seems to be associated with mortality in our patient group (fig 1). Recently published preliminary data on 40000 participants in UK Biobank further showed that adverse $M C$, identified using the same method as in this study, is associated with four times higher mortality in general population(13). These results, in combination with the results on the CKD patients in the present study, highlight that identification of adverse $M C$ can be used to understand mortality risk related to sarcopenia across disease stages, not only in end stage disease (fig 2).

Hypoalbuminemia is a well-known predictor of mortality in patients with CKD (25) and it has been reported that there is an association between sarcopenia and hypoalbuminemia $(24,26)$. In our study, we 
observed that patients in the non-survivor group (with a dominant adverse $M C$ ) had significantly lower serum albumin levels.

The strengths of this study include the detailed assessment of muscle composition (muscle volume and fat infiltration) with MRI and the use of quantitative muscle biomarkers with strong associations to functional performance and outcomes. The use of a highly standardized image acquisition protocol and systematic quality control procedures for muscle composition quantification enabled comparison between the study participants and virtual controls assessed in UK Biobank thus increasing the study size and enabling body size-independent sarcopenia assessment. The study has some limitations, including relatively low sample size and gender bias (only 2/11 of participants were women). A larger-scale study is needed to confirm our results.

\section{Conclusions}

This study demonstrates that this novel MRI method, combining body size-adjusted muscle volume and muscle fat infiltration, can be used to analyze muscle composition in CKD patients. Furthermore, MRI based muscle composition assessment did reflect comorbidity and could effectively predict survival in HD patients. The combined assessment including both muscle volume and muscle fat infiltration strengthened the predictive performance.

Our findings suggest that MRI based muscle composition assessment can be used for identification of sarcopenia in CKD patients where early therapeutic interventions may prevent or delay the progression of sarcopenia.

\section{Abbreviations}

MRI: Magnetic resonance imaging

CKD: Chronical kidney diseases

MFI: Muscle fat infiltration

Adverse MC: Adverse muscle composition

HD: Hemodialysis

nCl: New comorbidity index

FFMV: Fat-free muscle volume in the thighs

FFMV z-score: Fat-free muscle volume z-score.

Muscle $_{\text {comb }}$ :Combined muscle score 
MFI: Muscle fat infiltration

$\mathrm{MFI}_{\text {adj: }}$ a sex-adjusted MFI

$D M$ : Diabetes mellitus

\section{Declarations}

\section{ETHICS APPROVAL AND CONSENT TO PARTICIPATE}

The Regional Ethical Review Board in Linköping approved (2013/475-31) the study protocol, and written informed consent was obtained from all patients. All methods were performed in accordance with the relevant guidelines and regulations.

\section{CONSENT FOR PUBLICATION}

Not applicable.

\section{AVAILABILITY OF DATA AND MATERIALS}

The datasets generated and/or analyzed during the study are available from the corresponding author on reasonable request.

\section{AUTHOR INFORMATION}

Corresponding author:

Ainhoa Indurain. E-mail: Ainhoa.indurain@liu.se

Co-authors:

Jennifer Linge. E-mail: jennifer.linge@amramedical.com

Mikael Petersson. E-mail: mikael.petersson@amramedical.com

Thobias Romu. E-mail: thobias.romu@amramedical.com

Fredrik Uhlin. E-mail: fredrik.uhlin@regionostergotland.se

Anders Fernström. E-mail: anders.fernstrom@regionostergotland.se

Mårten Segelmark. E-mail: marten.segelmark@med.lu.se

Olof Dahlqvist Leinhard. E-mail: olof.dahlqvist.leinhard@amramedical.com

\section{AUTHORS' CONTRIBUTIONS}


M. Segelmark and O. Dahlqvist Leinhard were involved in the concept and design. A. Indurain, T. Romu were involved in the acquisition and analysis of data. A. Indurain drafted the manuscript. A.Indurain, J. Linge, F. Uhlin, A. Fernström, M. Segelmark and O. Dahlqvist Leinhard were responsible for interpretation of data and for critical revision of the manuscript for important intellectual content. M. Petersson was responsible for the statistical analysis.

\section{COMPETING INTERESTS}

Al, FU, AF, MS declared no competing interests. JL, TR and ODL are employees and stockholders of AMRA Medical AB. MP is an employee of AMRA Medical AB.

The results presented in this article have not been published previously in whole or part, except in abstract form.

\section{FUNDING}

Funding for image analysis of the UK Biobank data was gratefully received from Pfizer Inc.

\section{ACKNOWLEDGEMENTS}

A.Indurain thanks The Swedish Research Council, The Swedish Kidney Foundation and The Swedish Society of Nephrology for financial support.

\section{References}

1. Moorthi RN, Avin KG. Clinical relevance of sarcopenia in chronic kidney disease. Current opinion in nephrology and hypertension. 2017;26(3):219.

2. Cruz-Jentoft AJ, Bahat G, Bauer J, Boirie Y, Bruyère O, Cederholm T, et al. Sarcopenia: revised European consensus on definition and diagnosis. Age and ageing. 2019;48(1):16-31.

3. Souza VA, Oliveira D, Barbosa SR, Corrêa J, Colugnati FAB, Mansur HN, et al. Sarcopenia in patients with chronic kidney disease not yet on dialysis: Analysis of the prevalence and associated factors. PloS one. 2017;12(4):e0176230.

4. Moon SJ, Kim TH, Yoon SY, Chung JH, Hwang HJ. Relationship between Stage of Chronic Kidney Disease and Sarcopenia in Korean Aged 40 Years and Older Using the Korea National Health and Nutrition Examination Surveys (KNHANES IV-2, 3, and V-1, 2), 2008-2011. PloS one. 2015;10(6):e0130740.

5. Giglio J, Kamimura MA, Lamarca F, Rodrigues J, Santin F, Avesani CM. Association of Sarcopenia With Nutritional Parameters, Quality of Life, Hospitalization, and Mortality Rates of Elderly Patients on Hemodialysis. Journal of renal nutrition : the official journal of the Council on Renal Nutrition of the National Kidney Foundation. 2018;28(3):197-207. 
6. Kim JK, Kim SG, Oh JE, Lee YK, Noh JW, Kim HJ, et al. Impact of sarcopenia on long-term mortality and cardiovascular events in patients undergoing hemodialysis. The Korean journal of internal medicine. 2019;34(3):599-607.

7. Kato A. Epidemiology of Sarcopenia and Frailty in CKD. In: Kato A, Kanda E, Kanno Y, editors. Recent Advances of Sarcopenia and Frailty in CKD. Singapore: Springer Singapore; 2020. p. 1-14.

8. Linge J, Heymsfield SB, Dahlqvist Leinhard O. On the Definition of Sarcopenia in the Presence of Aging and Obesity-Initial Results from UK Biobank. The journals of gerontology Series A, Biological sciences and medical sciences. 2020;75(7):1309-16.

9. Beaudart $C$, McCloskey $E$, Bruyère $O$, Cesari $M$, Rolland $Y$, Rizzoli $R$, et al. Sarcopenia in daily practice: assessment and management. BMC geriatrics. 2016;16(1):170.

10. Linge J, Borga M, West J, Tuthill T, Miller MR, Dumitriu A, et al. Body Composition Profiling in the UK Biobank Imaging Study. Obesity (Silver Spring, Md). 2018;26(11):1785-95.

11. Heymsfield SB, Gonzalez MC, Lu J, Jia G, Zheng J. Skeletal muscle mass and quality: evolution of modern measurement concepts in the context of sarcopenia. The Proceedings of the Nutrition Society. 2015;74(4):355-66.

12. Linge J, Ekstedt $M$, Dahlqvist Leinhard $O$. Adverse muscle composition is linked to poor functional performance and metabolic comorbidities in NAFLD. JHEP reports : innovation in hepatology. 2021;3(1):100197.

13. Linge J, Petersson M, Forsgren MF, Sanyal AJ, Dahlqvist Leinhard O. Adverse muscle composition predicts all-cause mortality in the UK Biobank imaging study. Journal of cachexia, sarcopenia and muscle. 2021.

14. Liu J, Huang Z, Gilbertson DT, Foley RN, Collins AJ. An improved comorbidity index for outcome analyses among dialysis patients. Kidney international. 2010;77(2):141-51.

15. Kan WC, Wang JJ, Wang SY, Sun YM, Hung CY, Chu CC, et al. The new comorbidity index for predicting survival in elderly dialysis patients: a long-term population-based study. PloS one. 2013;8(8):e68748.

16. Kim KM, Jang HC, Lim S. Differences among skeletal muscle mass indices derived from height-, weight-, and body mass index-adjusted models in assessing sarcopenia. The Korean journal of internal medicine. 2016;31(4):643-50.

17. Mori K, Nishide K, Okuno S, Shoji T, Emoto M, Tsuda A, et al. Impact of diabetes on sarcopenia and mortality in patients undergoing hemodialysis. BMC nephrology. 2019;20(1):105.

18. Curcio F, Testa G, Liguori I, Papillo M, Flocco V, Panicara V, et al. Sarcopenia and Heart Failure. Nutrients. 2020;12(1).

19. Pacifico J, Geerlings MA, Reijnierse EM, Phassouliotis C, Lim WK, Maier AB. Prevalence of sarcopenia as a comorbid disease: A systematic review and meta-analysis. Experimental gerontology. 2020;131:110801.

20. Cebrià i Iranzo MA, Arnal-Gómez A, Tortosa-Chuliá MA, Balasch-Bernat M, Forcano S, SentandreuMañó $T$, et al. Functional and clinical characteristics for predicting sarcopenia in institutionalised 
older adults: Identifying tools for clinical screening. International journal of environmental research and public health. 2020;17(12):4483.

21. Gong G, Wan W, Zhang X, Liu Y, Liu X, Yin J. Correlation between the Charlson comorbidity index and skeletal muscle mass/physical performance in hospitalized older people potentially suffering from sarcopenia. BMC geriatrics. 2019;19(1):367.

22. Inaba M, Okuno S, Ohno Y. Importance of Considering Malnutrition and Sarcopenia in Order to Improve the QOL of Elderly Hemodialysis Patients in Japan in the Era of 100-Year Life. Nutrients. 2021;13(7):2377.

23. Pereira RA, Cordeiro AC, Avesani CM, Carrero JJ, Lindholm B, Amparo FC, et al. Sarcopenia in chronic kidney disease on conservative therapy: prevalence and association with mortality. Nephrology, dialysis, transplantation : official publication of the European Dialysis and Transplant Association uropean Renal Association. 2015;30(10):1718-25.

24. Wilkinson TJ, Miksza J, Yates T, Lightfoot CJ, Baker LA, Watson EL, et al. Association of sarcopenia with mortality and end-stage renal disease in those with chronic kidney disease: a UK Biobank study. Journal of cachexia, sarcopenia and muscle. 2021;12(3):586-98.

25. Kalantar-Zadeh K, Ikizler TA. Let them eat during dialysis: an overlooked opportunity to improve outcomes in maintenance hemodialysis patients. Journal of renal nutrition. 2013;23(3):157-63.

26. Oliveira EMd, Silva R, Lemos M, Burgos MGPdA, Costa DMN, Maio R. Frequency of sarcopenia, cachexia, and associated factors in patients with chronic kidney disease in dialysis treatment. Nutricion Hospitalaria. 2020 Dec 16;37(6):1157-1165

\section{Figures}




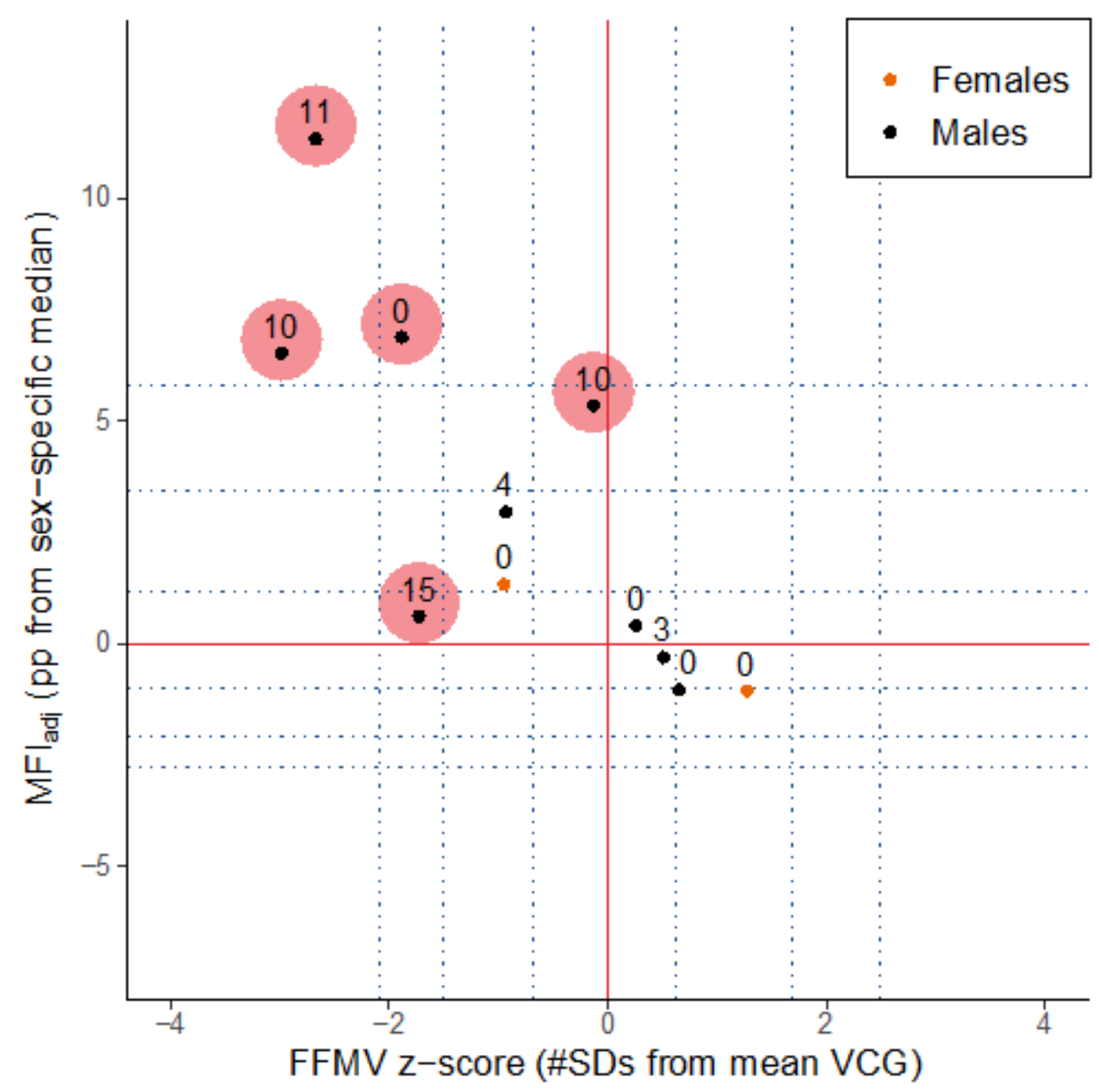

Figure 1

Distribution of FFMV z-score and $\mathrm{MFI}_{\text {adj }}$ among the patients in the dialysis dataset.

The number next to each observation is the $\mathrm{nCl}$ score. The five observations with red circles around correspond to deaths. The blue dotted lines show the 1st, 5th, 25th, 75th, 95th, and 99th percentile in the UK Biobank dataset for each variable. FFMV z-score :virtual control group-based fat free muscle volume. $\mathrm{MFI}_{\text {adj: }}$ muscle fat infiltration adjusted for sex. SD: standard deviation. VCG: virtual control group. 

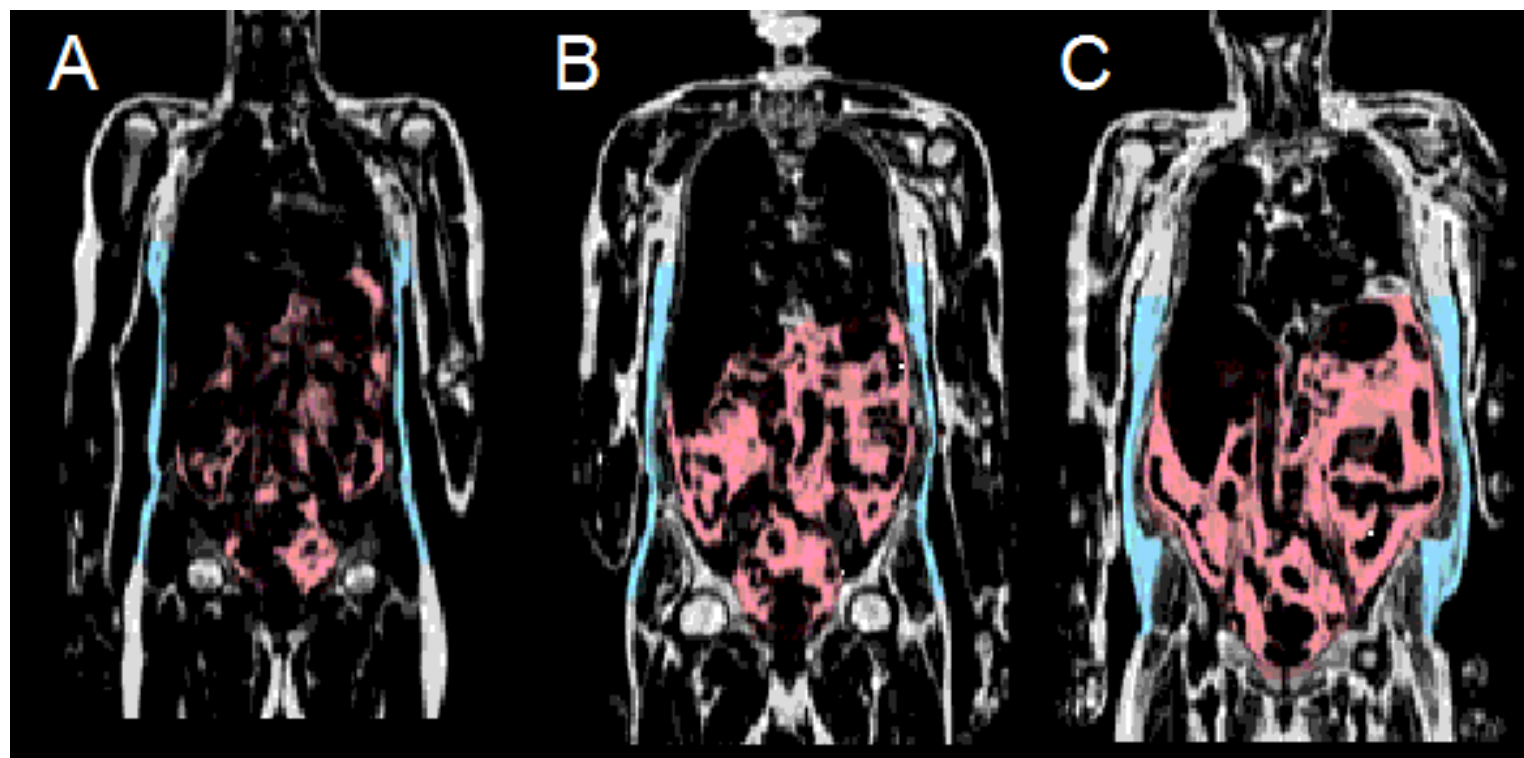

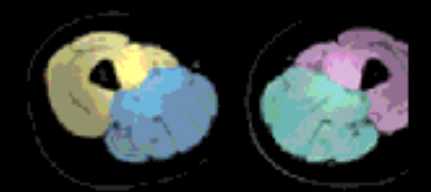

FFMV z-score: $1.3 \mathrm{SD}$ MFI: $6.6 \%$
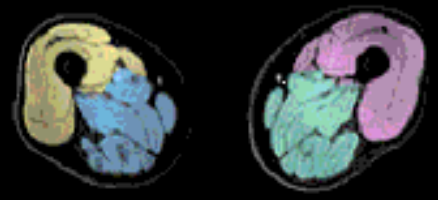

FFMV z-score: -0.9 SD MFI: $9.5 \%$
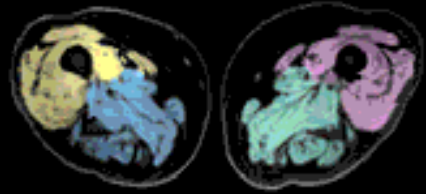

FFMV z-score: -2.7 SD MFI: $17.9 \%$

\section{Figure 2}

Coronal slice MRI scan and transversal slice with thigh muscle segmentations in 3 different patients

Patient A with normal muscle composition. Patients $B$ and $C$ with adverse muscle composition, most notable in the patient C. FFMV z-score: virtual control group-based fat free muscle volume. SD: standard deviation. MFI: muscle fat infiltration. 